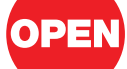

SUBJECT AREAS: BIOFUELS

CARBOHYDRATE CHEMISTRY

RENEWABLE ENERGY

CATALYST SYNTHESIS

Received

9 November 2012

Accepted

14 November 2012

Published

7 February 2013

Correspondence and requests for materials should be addressed to Y.F. (fuyao@ustc.edu.

$\mathrm{cn})$

\section{Linked strategy for the production of fuels via formose reaction}

\author{
Jin Deng, Tao Pan, Qing Xu, Meng-Yuan Chen, Ying Zhang, Qing-Xiang Guo \& Yao Fu
}

Anhui Province Key Laboratory of Biomass Clean Energy, Department of Chemistry, University of Science and Technology of China, Hefei 230026, China.

Formose reaction converts formaldehyde to carbohydrates. We found that formose reaction can be used linking the biomass gasification with the aqueous-phase processing (APP) to produce liquid transportation fuel in three steps. First, formaldehyde from syn-gas was converted to triose. This was followed by aldol condensation and dehydration to 4-hydroxymethylfurfural (4-HMF). Finally, 4-HMF was hydrogenated to produce 2,4-dimethylfuran (2,4-DMF) or $\mathrm{C}_{9}-\mathrm{C}_{15}$ branched-chain alkanes as liquid transportation fuels. In the linked strategy, high energy-consuming pretreatment as well as expensive and polluting hydrolysis of biomass were omitted, but the high energy recovery of APP was inherited. In addition, the hexoketoses via formose reaction could be converted to HMFs directly without isomerization. A potential platform molecule 4-HMF was formed simultaneously in APP.

ontinuously increasing oil prices along with growing concerns about emission of greenhouse gas have
made the renewable liquid fuels a focus of attention ${ }^{1-6}$. Among all the efforts to search for alternative fuel
sources, biomass has been considered to be "the only practical source of renewable liquid fuel"7. Currently, there are three main routes to make fuels from biomass: (i) pyrolysis to make bio-oils followed by zeolite upgrading or hydrodeoxygenation to liquid fuels; (ii) gasification to produce syn-gas followed by FischerTropsch synthesis (FTS) to alkanes; and (iii) acid hydrolysis to give sugars followed by aqueous-phase processing (APP) to high-quality liquid fuels ${ }^{5}$. Among these strategies, the APP converts biomass-derived sugars into $\mathrm{H}_{2}$ or high-quality liquid fuels and it has benign reaction conditions and energy recovery as high as $96 \%$. The APP was developed by Dumesic and co-workers and is believed to be a promising route for industry-scale production ${ }^{8}$. However, the pretreatment and acid hydrolysis of woody biomass to sugars are costly and may cause environmental concerns. According to National Renewable Energy Laboratory's estimation, the cost of unrefined sugar monomers in aqueous solution produced from lignocellulose is as high as $12-14 € / \mathrm{kg}_{\text {sugar }}{ }^{9}$. Moreover, APP cannot use entire organic matter in lignocellulose ${ }^{5}$. In contrast, the gasification route, which is a well established technology, can convert the entire biomass into syn-gas and its process thermal efficiency (PTE) is $77 \%{ }^{10}$. However, during the conversion of syn-gas into liquid fuels by FTS, a large amount of energy is lost and the overall PTE is only $16-43 \%{ }^{10}$. In this respect, a low-cost and high-efficiency refinery technology still remains absence.

Herein, we outline a linked strategy which links the gasification and the APP via formose reaction. As shown in Fig. 1, woody biomass was completely converted into syn-gas at first. Syn-gas was subsequently converted into sugars via linkage reactions and then underwent APP via 4-hydroxymethylfurfuran (4-HMF) platform to produce 2,4-dimethylfuran (2,4-DMF) or $\mathrm{C}_{9}-\mathrm{C}_{15}$ branched-chain alkanes as liquid transportation fuels. In the linkage reactions, methanol synthesis has a highest PTE of $85 \%$ from syn-gas and could provide additional heat ${ }^{10}$; the matured production of formaldehyde from methanol has carbon yield of $92 \%$ with the theoretical PTE of $94 \%$ and could provide additional heat and hydrogen; the formose reaction has carbon yield of $96 \%$ and the theoretical PTE of $91 \%$ under mild reaction conditions. Thus, all of the linkage reactions provide excellent energy efficiencies and carbon efficiencies. Therefore, the linked strategy retains the advantages yet avoids the shortcomings of both gasification-FTS and hydrolysis-APP routes. It should be noted that, in one hand, as another industrial process, methanol to gasoline (MTG) has high energy efficiency of about 92-93\%, but MTG is unable to directly produce diesel and jet fuel which cannot be produced by the zeolite catalyst (ZSM-5) used in this process ${ }^{11}$. Furthermore, MTG has poor market control ability due to lacking of multifunctional intermediates. In another hand, combined gasification-biological processing is another alternative, such as microbial conversion of syn-gas into ethanol. However, this process has its inherent shortcomings, namely, water-solubility and has low calorific value of ethanol. 


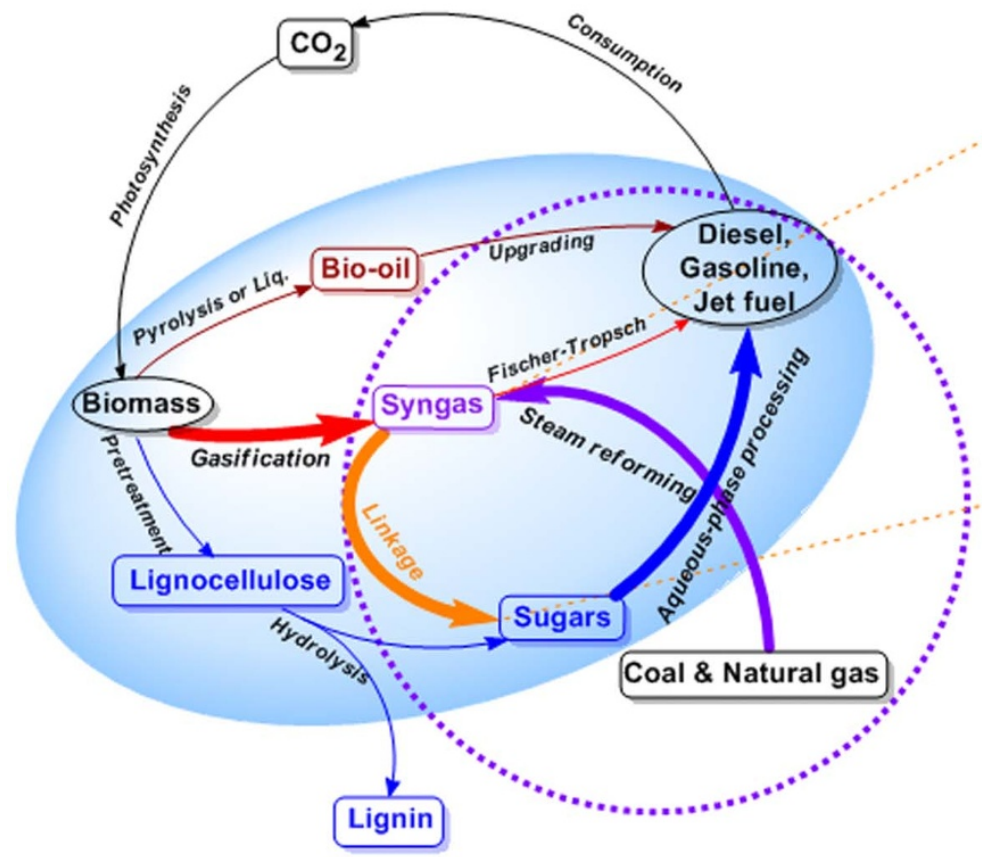

$$
\begin{aligned}
& \text { Methanol Production } \\
& \mathrm{CO}_{2}+3 \mathrm{H}_{2}=\mathrm{CH}_{3} \mathrm{OH}+\mathrm{H}_{2} \mathrm{O} \\
& \mathrm{CO}+2 \mathrm{H}_{2}=\mathrm{CH}_{3} \mathrm{OH} \\
& \text { Fromaldehyde Production } \\
& \mathrm{CH}_{3} \mathrm{OH}+1 / 2 \mathrm{O}_{2}=\mathrm{HCHO}+\mathrm{H}_{2} \mathrm{O} \\
& \mathrm{CH}_{3} \mathrm{OH}=\mathrm{HCHO}+\mathrm{H}_{2}
\end{aligned}
$$

Formose Reaction

$3 \mathrm{HCHO}=\mathrm{C}_{3} \mathrm{H}_{6} \mathrm{O}_{3}$
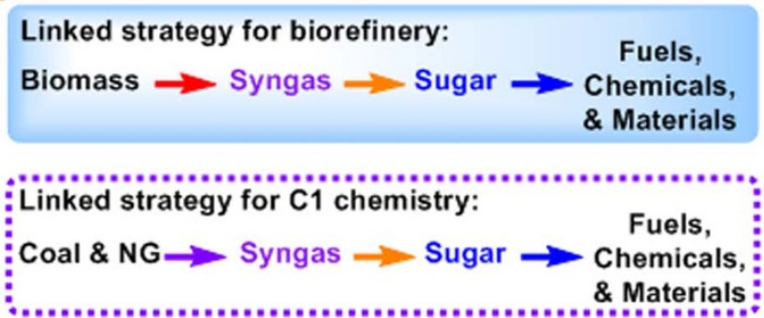

Figure $1 \mid$ Linked strategy links the gasification and the APP via formose reaction. Routes are for the biorefinery (highlight with cyan ellipse) and for the $\mathrm{C} 1$ chemistry (highlight with purple dotted cycle). Brown arrows refer to pyrolysis-upgrading route (thermolysis strategy), red arrows refer to gasification-FTS route (thermolysis strategy), blue arrows refer to hydrolysis-APP route (hydrolysis strategy), purple arrow refers to gasification of coal or reforming of nature gas ( $\mathrm{C} 1$ chemistry), orange arrow linking the syn-gas and sugars refers to the linkage reactions including methanol production, formaldehyde production and formose reaction (highlight with orange rectangle), and black arrows refer to carbon recycle. The bold red arrow, bold orange arrow and bold blue arrow make up the linked strategy for biorefinery (highlight with cyan rectangle). The bold purple arrow, bold orange arrow and bold blue arrow make up the linked strategy for $\mathrm{Cl}$ chemistry (highlight with purple dotted rectangle).

The linked strategy to produce liquid fuel assigns another importance of overcoming the energy crisis to the classic formose reaction. Although the linked strategy has relatively long processes, it has $c a$. 1.2 times energy recovery and $c a$. twice liquid fuels yield compared with gasification-FTS route. Most importantly, not only the carbon dioxide can be used as the reaction material but also value-added chemicals can be produced as the reaction intermediates. In comparison with the hydrolysis-APP route, the linked strategy achieves the entire use of biomass, and avoids the high energy-consuming pretreatment as well as expensive and polluting hydrolysis. Moreover, rare branched-chain ketohexose was produced. It may not only form furan structure by direct dehydration without isomerization $^{5,12-15}$, but also form 4 -HMF with a 2,4-substituted furan structure which is difficult to synthesize before ${ }^{16,17}$. As a potential platform molecule, 4-HMF shows broad application prospects. In this report, we not only demonstrated the possibility for the production of a renewable liquid fuel 2,4-DMF from 4-HMF, but also prepared high-octane fuel of $\mathrm{C}_{9}-\mathrm{C}_{15}$ branched-chain alkanes. In addition, the linked strategy can also utilize the syn-gas produced from the gasification of coal or the reforming of natural gas and thus expand the repertoire of $\mathrm{C} 1$ chemistry.

\section{Results}

Figure 2 illustrates our approach to convert methanol to 2,4-DMF as liquid transform fuel. First, biomass derived methanol was obtained via gasification and methanol synthesis, and subsequently converted into formaldehyde. Currently, as the largest downstream market of methanol, $35 \%$ worldwide methanol is consumed to produce formaldehyde through two mainstream technologies: the BASF process with silver catalyst, and the Formox process with metal oxide catalyst ${ }^{10}$. In the BASF process which is presently more economic (production cost is $0.38 \$ / \mathrm{kg}$ ) and widely adopted, the dehydrogenation and partial oxidation of methanol proceed simultaneously as following:

$$
\begin{aligned}
& \mathrm{CH}_{3} \mathrm{OH} \rightarrow \mathrm{HCHO}+\mathrm{H}_{2} \text { (dehydrogenation) } \Delta \mathrm{Hr}=+84 \mathrm{~kJ} / \mathrm{mol} \\
& \mathrm{CH}_{3} \mathrm{OH}+1 / 2 \mathrm{O}_{2} \rightarrow \mathrm{HCHO}+\mathrm{H}_{2} \mathrm{O} \text { (partial oxidation) } \\
& \Delta \mathrm{Hr}=-159 \mathrm{~kJ} / \mathrm{mol}
\end{aligned}
$$

In a typical production, between 50 and $60 \%$ formaldehyde was formed via partial oxidation, and the total yield of formaldehyde is about $92 \%$. The net result provides additional heat and hydrogen ${ }^{18}$.

Next, to achieve a cost-effective production of liquid fuel from formaldehyde with the potential for commercialization, we designed an integrated continuous process. In details, we carried out the selfcondensation of paraformaldehyde catalyzed by thiazolium salts reported by Matsumoto et al. ${ }^{19}$ to produce 1,3-dihydroxyacetone (DHA) in high selectivities and yields. From the economical point of view, formalin (an aqueous solution of formaldehyde) is a better feedstock than anhydrous paraformaldehyde. Considering that the thiazolium-based catalysts are immediately deactivated in the presence of water and this deactivation could be released by removing the water, an ingenious process designed by Avison et al. can be used for reference ${ }^{20,21}$. It was reported that by using this process, $96 \%$ selectivity was achieved while the single-pass conversion was $c a$. $30 \%$ (see fig.S10 for details about continuous process).

As a triose, the carbon number of DHA is not suitable as a feedstock to produce liquid fuels. Noting that the aldol condensation reaction of ketones is a classic method to increase the carbon chain length in organic chemistry, and features several advantages such as high efficiency, high carbon recovery and high energy recovery, we subsequently investigated the aldol self-condensation of DHA to produce hexoses. In a typical aldol condensation reaction, a strong basic condition is required. We screened four commercial strong basic ionic exchange resins, and selected IRA-900 $\left(\mathrm{OH}^{-1}\right)$ as the best catalyst. Theoretically, the aldol condensation of DHA would generate a branched-chain ketohexose (dendroketose), but a small amount of straight-chain ketohexoses including fructose and sorbose were also produced. To interpret the observations, we proposed 


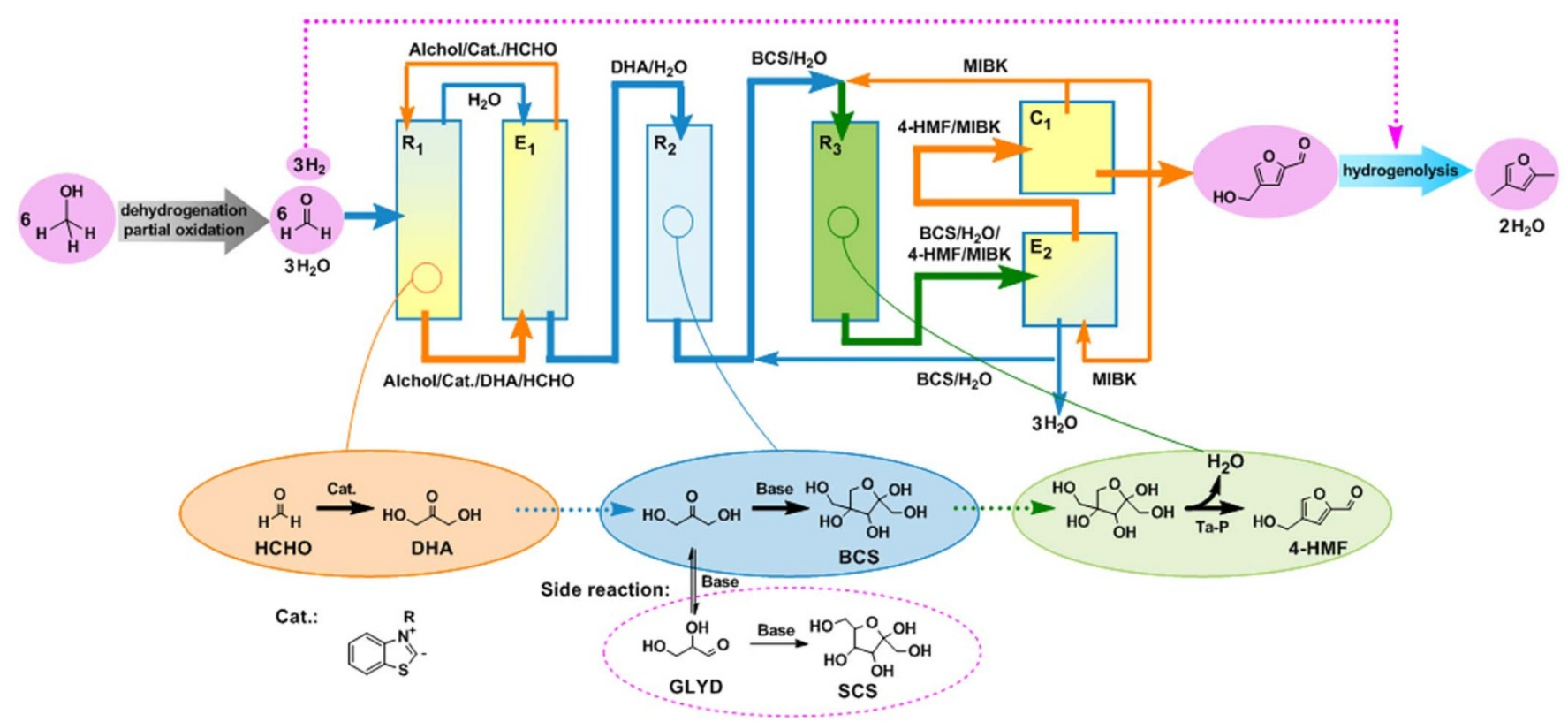

Figure $2 \mid$ Schematic diagram of the process for conversion of methanol to 2,4-dimethylfuran. Diagram includes selective condensation of formaldehyde which convert from methanol to form dihydroxyacetone (DHA) in a condensation reactor (R1); separation aqueous solution of DHA from the organic solvents and catalyst (E1); condensation of the aqueous solution of DHA to dendroketose over a strong-basic ionic resin (R2); selective dehydration of dendroketose to form 4-HMF in a biphasic mixture continuous reactor (R3); separation of the 4-HMF from the unreacted intermediates in a continuous countercurrent extractor (E2); concentration the organic solvent containing 4-HMF and recycle of extracting solvent, leading to separation of 4-HMF (C1); and hydrogenolysis of 4-HMF to 2,4-DMF. See text for details.

that DHA could isomerize to glyceraldehyde (GLYD) under basic conditions, and the subsequent condensation of GLYD with DHA could produce straight-chain ketohexoses ${ }^{22,23}$. The ratio of produced branched-vs. straight-chain sugars was closely related to the reaction temperature. The branched vs. straight chain sugar ratio dropped from $c a .19$ to $c a .8$ as temperature increased from $273 \mathrm{~K}$ to $303 \mathrm{~K}$, which could be explained by the promotion of isomerization at higher temperatures. Interestingly, the branched vs. straight chain sugar ratio has an obvious distinction with Ref. 23. The ${ }^{13} \mathrm{C}-\mathrm{NMR}$ experiments further proved the ratio (details in SI). It should be noted that under higher temperatures, the overall sugar yield also decreased, which is due to that DHA and GLYD can isomerize to lactic acid and then irreversibly converted into lactate ${ }^{24,25}$. Therefore, we selected $273 \mathrm{~K}$ as the optimal temperature, and conducted experiments of the continuous aldol condensation of DHA. The experiments showed that nearly $100 \%$ of ketohexoses yields were achieved at liquid hourly space velocity (LHSV) below $1 \mathrm{~h}^{-1}$. With the further increase of LHSV, the sugar yield dropped obviously, and incomplete conversion of DHA was detected. Interestingly, the selectivity of branched-chain sugar increased with the increase of LHSV. This could be explained that the production of branched chain sugar does not require the isomerization step and therefore has a faster reaction rate (see table S1, fig.S11 and relate text in Supplementary Information about continuous process).

Unlike hexoaldoses (e.g. glucose) which need to isomerize into hexoketoses before the dehydration to form the furan structure $\mathrm{e}^{12-15}$, dendroketose can be directly dehydrated into 4-HMF in high boiling point organic solvents catalyzed by sulfonic acid resins. Our experiment using DMSO as solvent showed over 90\% yield of 4 -HMF. However, the separation of 4-HMF in this process is difficult and results in high energy consumption. In aqueous solutions, the dehydration of dendroketose is nonselective and produces large amount of by-products besides 4-HMF. Considering the successful application of biphasic system in the production of $5-\mathrm{HMF}^{26}$ and the high selectivity in the conversion of fructose into 5 - $\mathrm{HMF}$ catalyzed by tantalum compounds ${ }^{27}$, we investigated the dehydration of dendroketose in biphasic system catalyzed by tantalum-based solid acid catalyst in a continuous fixed-bed reactor. Under a fixed LHSV of $1 \mathrm{~h}^{-1}$, the selectivity of 4-HMF slightly increased with the higher feed ratio of organic solvent (methyl isobutyl ketone, MIBK) to aqueous dendroketose solution. The space velocity also significantly affected the selectivity of 4-HMF. With the feed ratio fixed at 2:1 (org:aq), the lower LHSV led to a higher conversion of dendroketose but a rapid drop of 4-HMF selectivity, while the increase of LHSV resulted in a lower conversion but a higher selectivity (see table S2, fig. S12 and relate text in Supplementary Information about continuous process).

Combining the process in Fig. 2 together, we have achieved the continuous process from formaldehyde into 4-HMF. Under optimized conditions, the production of DHA from formaldehyde has a selectivity of $96 \%$ with a single-pass conversion of ca. $30 \%$; the condensation of DHA into dendroketose achieved $100 \%$ conversion and $>99 \%$ ketohexose selectivity (branched-chain ketohexose : straight-chain ketohexoses $=94: 6$ ); the dehydration of aqueous dendroketose into $4-\mathrm{HMF}$ achieved a selectivity of $96 \%$ with a singlepass conversion of $71 \%$. Theoretically, the overall process has a carbon efficiency of $100 \%$ and the PTE of $92 \%$, with water as the only by-product. At this point, we have demonstrated the feasibility of the production of a potential platform molecule 4-HMF. Because 4-HMF has the same functional group and similar structure with 5-HMF, branched-chain alkanes with the number of carbon atoms ranging from $\mathrm{C}_{9}$ to $\mathrm{C}_{15}$ can be produced from 4-HMF via aqueousphase processing developed by Dumesic and co-workers ${ }^{8}$.

The feasibility from 4-HMF to $\mathrm{C}_{9} \sim \mathrm{C}_{15}$ branched-chain alkanes was demonstrated and a similar process was conducted according to Ref. 8. After condensation of 4-HMF with acetone and hydrogenation of condensation products the step of hydrodeoxygenation was studied (Fig. 3). The catalyst of Pt/C and HZSM-5 was employed. The results showed that the molar yield of alkanes gradually increased with reaction temperature, but the carbon atoms number of the main products decreased from $\mathrm{C}_{13}-\mathrm{C}_{14}$ to $\mathrm{C}_{12}$ due to $\mathrm{C}$ - $\mathrm{C}$ cleavage in higher temperature under platinum catalyst. Considering the high activity of supported Pt catalyst and to reduce the loss of carbon 


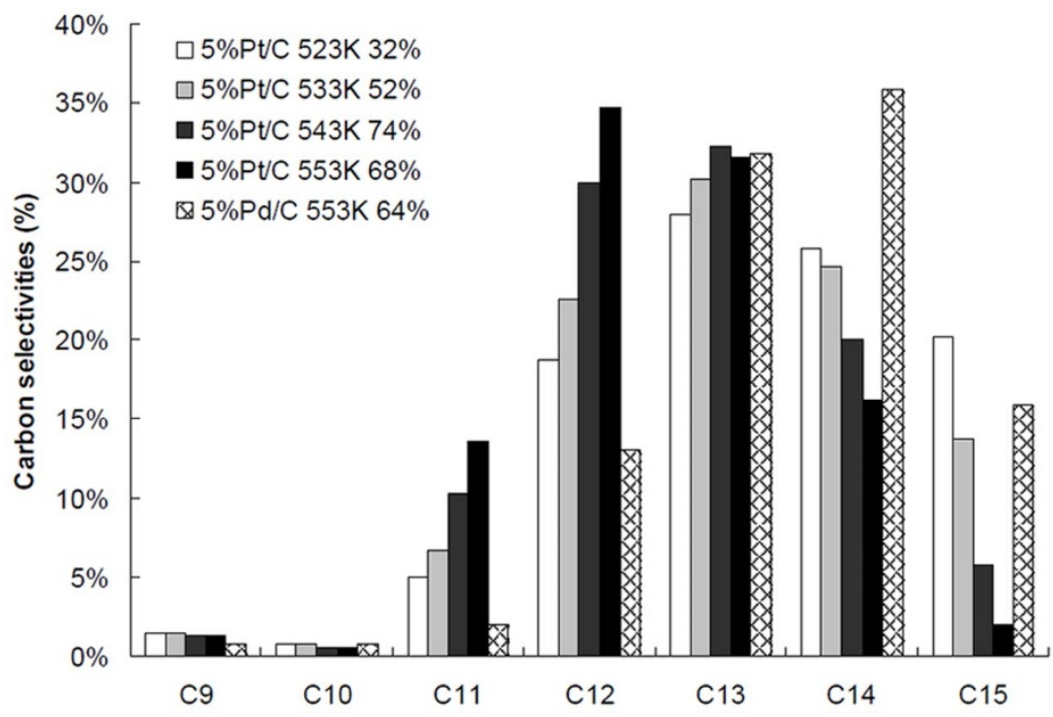

Figure 3 Carbon selectivities from hydrodeoxygenation processing of various reaction temperature and catalysts.

atoms, Pd/C and HZSM-5 was used. Surprisingly, a moderate yield $(64 \%$ at $553 \mathrm{~K})$ and the carbon atoms number (mainly $\mathrm{C}_{14}$ ) of branched-chain alkanes were achieved (see GC chromatogram in $\mathrm{SI})$. Notably, when $\mathrm{Pt} / \mathrm{C}$ was performed without additive of HZSM-5, a half yield of alkane $(36 \%$ at $543 \mathrm{~K})$ was obtained due to the lack of acidity of HZSM-5 to deoxygenate.
Note that it is a good strategy to maximize the advantage of reaction intermediates and balance the high-value/low-volume products with high-volume/low-value fuels ${ }^{28}$. 4 -HMF is a compound with a rare $\beta$-substituted furan structure which can be converted into 2,4$\mathrm{DMF}$ as a liquid fuel with high energy-density/low water solubility via high-value intermediates such as 2 and 3 (Fig. 4). Dumesic and

a
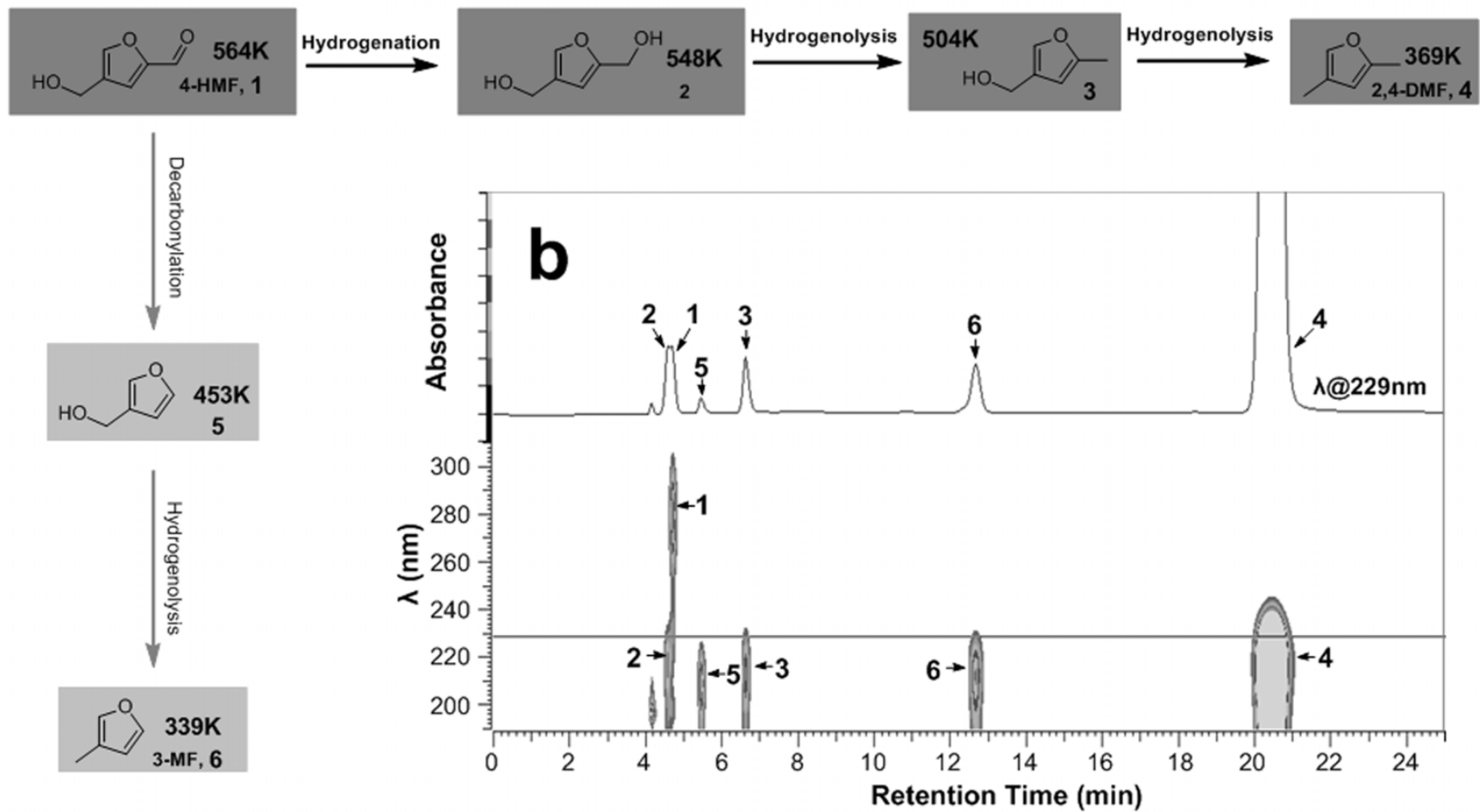

Figure $4 \mid \boldsymbol{\beta}$-substituted furans compounds formed by removal of oxygen atoms from 4-HMF. (a) Reaction pathways for conversion of 4-HMF (1) to 2,4-DMF (4) through 2,4-furandimethanol (2) and 2-methyl-furan-4-methanol (3) via hydrogenation and hydrogenolysis (black arrows), or to 3-methylfuran (6) through furan-3-methanol (5) via decarbonylation and hydrogenolysis (grey arrows). 2,4-tetrahydrofurandimethanol (7),

2,4-dimethyltetrahydrofuran (8) and 3-methyltetrahydrofuran (9) are the byproducts and byproduct intermediates of hydrogenation of the furan ring. Dark grey boxes highlight reactant, product and primary intermediates. Light grey boxes highlight key byproducts and byproduct intermediates. (b) High performance liquid chromatography of reactant (1), product (4), primary intermediates (2 and 3), key byproduct (5) and key byproduct intermediate (6). According to the different retention times and different maximum absorption wavelength, we can qualitative analyze these compounds. 
Table 1 | Hydrogenolysis of 4-HMF

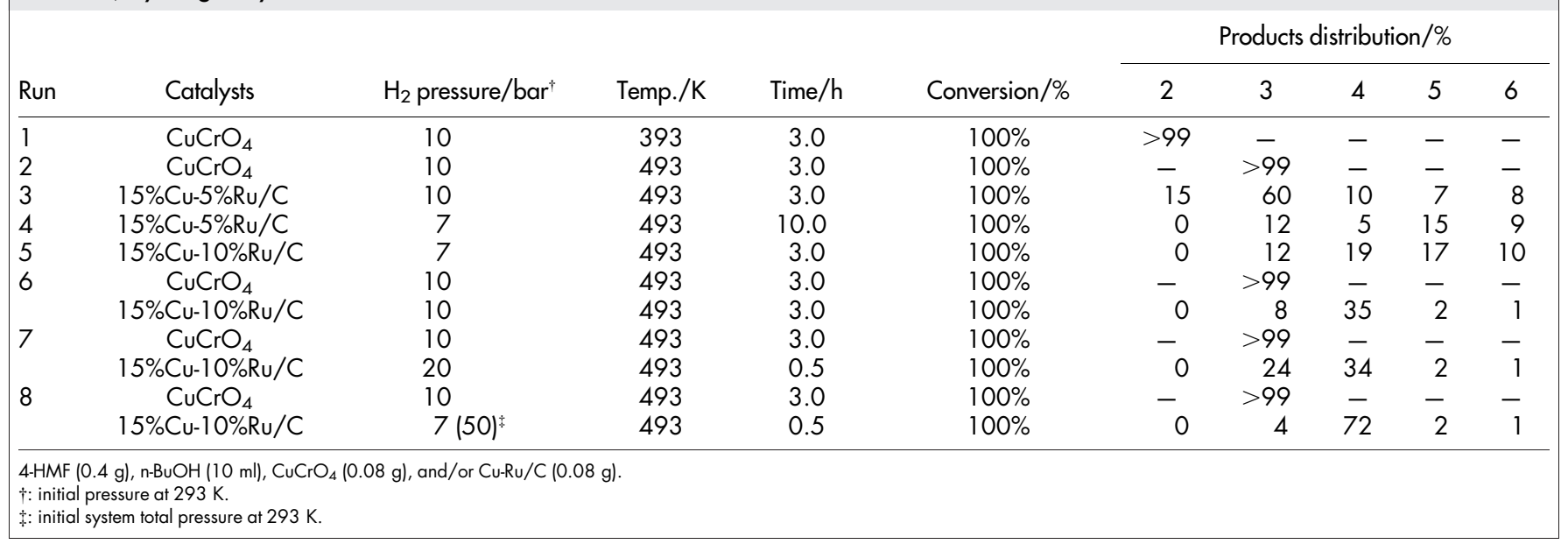

co-workers have developed a neat process in which 5-HMF is directly hydrogenated into $2,5-\mathrm{DMF}^{29}$. Taking this as a reference, we have developed a catalytic process for the production of 2,4-DMF from 4-HMF.

Previous studies show that $\mathrm{CuCrO}_{4}$ selectively converts furfural into furfuryl alcohol and 2-methylfuran ${ }^{30}$, and 5-HMF into 5methylfurfuryl alcohol and 2,5-DMF ${ }^{29}$. However, the hydrogenation of 4 -HMF, which has a structure of $\beta$-substituted furanmethanol, remains a challenge. Table 1 summarizes the results of the liquidphase hydrogenation of 4-HMF. Employing $\mathrm{CuCrO}_{4}$ as catalyst, $>99 \%$ compound 2 was yielded at $393 \mathrm{~K}$, whereas $>99 \%$ compound 3 was yielded at $493 \mathrm{~K}$. However, the $\beta$-hydroxymethyl could not be reduced into methyl. When using $\mathrm{Cu}-\mathrm{Ru} / \mathrm{C}$ catalyst, the $\beta$ hydroxymethyl could be reduced and 2,4-DMF was obtained, despite the yield was only $10 \%$. Extending the reaction time resulted in a drop of 2,4-DMF yield, due to the increase of hydrogenation of the furan ring or ring decomposition products. Increase of the loading of $\mathrm{Ru}$ gave a 2,4-DMF yield of 19\%, but significant increase of byproducts 5 and $\mathbf{6}$ were detected due to the activation of carbonyl group by $\mathrm{Ru}$. To suppress the decarbonylation reaction and reduce the loss of carbon, we developed a two-step method. 4-HMF was firstly converted into compound 3 with high selectivity by $\mathrm{CuCrO}_{4}$ catalyst. Subsequently, $\mathrm{Cu}-\mathrm{Ru} / \mathrm{C}$ was employed to hydrogenate the $\beta$-hydroxymethyl. By this method, the decarbonylation was efficiently inhibited, and the 2,4-DMF yield was improved to $35 \%$. Increasing the pressure of hydrogen in step 2 and shortening the reaction time led to incomplete conversion of compound 3, but an increase in 2,4-DMF selectivity. Based on this phenomenon, we used low pressure hydrogen and increasing the system pressure by filling $\mathrm{CO}_{2}$, and successfully achieved the conversion of 4 -HMF into 2,4-DMF as liquid fuel with a yield of $72 \%$.

\section{Discussion}

The linked strategy reported here for conversion of formaldehyde to 2,4-DMF or $\mathrm{C}_{9}-\mathrm{C}_{15}$ branched-chain alkanes as liquid fuels utilizes formose reaction to synthesis fuels based on 4-HMF. This strategy not only combined the advantages of gasification-FTS route and hydrolysis-APP route, but also is important because the hexoketoses from formaldehyde could be easily converted to HMFs without isomerization which is the biggest obstacle in the development of sustainable HMF-based economy in an industrial scale. We have built an continuous process to convert formalin to 4 -HMF and demonstrated the feasibility of producing 2,4-DMF or $\mathrm{C}_{9}-\mathrm{C}_{15}$ branchedchain alkanes from 4-HMF. Although challenges remain for industrial application, this study opens a new path for the biomass to liquid transportation fuel via formose reaction and put forwards a new type of voluminously available platform molecule, 4-HMF.

\section{Methods}

Conversion of formaldehyde to dihydroxyacetone (DHA). Preparation of Catalysts: The thiazolium ylide catalysts were prepared by reacting $\mathrm{N}$-alkylthiazolium halides or $\mathrm{N}$-alkylbenzothiazolium halides with the corresponding organic base under nitrogen. All chemicals were purchased from Alfa Aesar. For example 3-hexylbenzothiazolium bromide $(5.0 \mathrm{~g}, 16.7 \mathrm{mmol})$, triethylamine $(2.3 \mathrm{ml}$, $16.7 \mathrm{mmol}$ ) and dioxane $(50 \mathrm{ml})$ were heated at $353 \mathrm{~K}$ under nitrogen and stirred for $12 \mathrm{~h}$. After cooling, the precipitated triethylammonium bromide was filtered. The filtrate was called catalyst solution.

Condensation Reaction: Paraformaldehyde $(50 \mathrm{~g}, 1.67 \mathrm{~mol}$ as formaldehyde) and dioxane $(450 \mathrm{ml})$ were heated to $373 \mathrm{~K}$ under nitrogen and then catalyst solution $(50 \mathrm{ml})$ was added. The mixture was stirred at $373 \mathrm{~K}$ for $1 \mathrm{~h}$. After the prescribed time the reaction was quenched in ice water. The reaction mixture was analyzed by HPLC (analytical methods in SI). Analysis of the reaction mixture showed that the yield of DHA is $85 \%$ and the conversion of formaldehyde is $99 \%$. The reaction mixture was evaporated to remove the solvent. The residue was poured into water $(100 \mathrm{ml})$ and extracted with dichloromethane $(100 \mathrm{ml})$ three times to recycle the catalyst. The aqueous solution could be used directly in the following step or isolated to white powder of DHA dimer by recrystallization with ethanol and acetone. Detail experiments about continuous process for conversion of formaldehyde to DHA see reference 20,21 and fig. S10.

Conversion of DHA to hexoses. Batch process: $5 \mathrm{ml}$ of DHA aqueous solution $(10 \mathrm{wt} \%)$ and Amberlite ${ }^{\circledR}$ IRA-900 basic ion exchange resin $(0.5 \mathrm{~g})$ were stirred at setting temperature for $12 \mathrm{~h}$. After filtration to remove the resin, the filtrate was analyzed by HPLC. Experimental data see Table S1.

Continuous process: A fixed bed reactor with an inner diameter of $10 \mathrm{~mm}$ and a height of $382 \mathrm{~mm}$, equipped with an ethanol jacket for cooling was used for conversion of DHA to hexoses. The Amberlite ${ }^{\circledR}$ IRA-900 basic ion exchange resin was packed in the reactor and $10 \mathrm{wt} \%$ DHA aqueous solution was fed at a constant flowrate through the reactor at $273 \mathrm{~K}$. The liquid effluent was collected for quantitative analysis by HPLC. The aqueous solution could be used directly in the following step or isolated to white powder of dendroketose by recrystallization with ethanol and acetone. Experimental data see Table S1.

Conversion of dendroketose to 4-HMF. Catalyst Preparation and Characterization: The tantalum-based solid acid catalyst was prepared according to 27 . The catalyst tantalum phosphate (TA-p) was prepared by adding $100 \mathrm{~g}$ of tantalum hydroxide $\left(\mathrm{Ta}_{2} \mathrm{O}_{5} \cdot n \mathrm{H}_{2} \mathrm{O}\right.$, Ta sample, HKXC (China)) in $1500 \mathrm{ml}$ of $1 \mathrm{M} \mathrm{H}_{3} \mathrm{PO}_{4}$ solution and stirring at room temperature for $52 \mathrm{~h}$. The reaction mixture was aged at room temperature for $12 \mathrm{~h}$. The precipitate was filtered and washed 5 times with deionized water until the $\mathrm{pH}$ was neutral and dried overnight at $333 \mathrm{~K}$ in drying oven, followed by drying at $383 \mathrm{~K}$ in vacuum oven for $3 \mathrm{~h}$. Then the catalyst was calcined in air to $573 \mathrm{~K}$ with slow heating rate (at $1 \mathrm{~K} / \mathrm{min}$ ) and held at this temperature for $3 \mathrm{~h}$.

The surface areas of the catalyst were determined by Brunauer-Emmett-Teller (BET) isotherms of $\mathrm{N}_{2}$ adsorption at $77 \mathrm{~K}$. The X-ray diffraction (XRD) pattern of the TA-p was conducted with an X'pert (PANalytical) diffractometer operated at $60 \mathrm{kV}$ and $55 \mathrm{~mA}$, using $\mathrm{Ni}$-filtered $\mathrm{Cu}-\mathrm{K} \alpha$ radiation. FTIR spectra were recorded on a Fourier transform instrument Nicolet 8700 (Thermo Electron). Thermal analysis (TG-DSC, Thermogravimetric-Differential Scanning Calorimeter) of TA-p was measured with a SDT Q600 (TA Instruments) thermal analyzer. The catalyst was heated in flowing $\mathrm{N}_{2}$ from room temperature to $1073 \mathrm{~K}$ at rate of $10 \mathrm{~K} / \mathrm{min}$. 
Batch process 1: The dendroketose $(0.5 \mathrm{~g}, 2.8 \mathrm{mmol})$ was dissolved in DMSO $(2.5 \mathrm{ml})$, to which was added Amberlyst ${ }^{\circledR}-15$ acidic ion exchange resin $(0.25 \mathrm{~g})$. This mixture was stirred at $383 \mathrm{~K}$ for 5 hours and then cooled to room temperature and filtered. The product mixture was diluted with a known mass of deionized water, centrifuged to precipitate insoluble products, and analyzed with HPLC.

Batch process 2: A biphasic reaction system was employed to convert of dendroketose to 4-HMF. A mixture of dendroketose $(2.4 \mathrm{~g}, 13.3 \mathrm{mmol})$ and TA-p $(240 \mathrm{mg})$ in water $(24 \mathrm{ml})$ and MIBK $(36 \mathrm{ml})$ was introduced in a $100 \mathrm{ml}$ zirconium magnetic coupling drive high-pressure batch autoclave. Then, the reactor was sealed and flushed with $5 \mathrm{MPa}$ of $\mathrm{N}_{2}$. The reaction mixture was heated to $453 \mathrm{~K}$ and stirred at a rate of $1000 \mathrm{rpm}$ for $2 \mathrm{~h}$. After reaction, the reactor was cooled in ice water and depressurized carefully. After filtration to remove the catalyst, the filtrate was analyzed by HPLC described above. Dendroketose conversion and 4-HMF yield were calculated from the product of the aqueous and organic phase concentration determined by HPLC and their corresponding volumes after reaction because the value of $\mathrm{V}_{\text {org }} / \mathrm{V}_{\mathrm{aq}}$ changed after reaction. Analysis of the reaction mixture showed that the yield of 4 -HMF is $80 \%$ and the conversion of dendroketose is $99 \%$. It is known that the addition of inorganic salt (e.g., $\mathrm{NaCl}$ ) to the biphasic reaction system could promote the phase separation of aqueous phase and organic phase. But when the salt (e.g., $\mathrm{NaCl}, \mathrm{Na}_{2} \mathrm{SO}_{4}$ ) was added to the reaction mixture, the yield of 4-HMF decreased to $53 \%$ and $33 \%$ correspondingly because of decrease of catalytic activity due to the ion exchange with the catalyst TA-p.

Continuous process: The continuous process for conversion of dendroketose to 4-HMF was carried out in a down-flow fixed bed reactor. The tantalum-based solid acid catalyst TA-p was pelleted as follows: TA-p (100 g) was mixed with sesbania gum $(1 \mathrm{~g})$ in water and dried overnight at $333 \mathrm{~K}$ in drying oven. The mixture was tableted and calcined in air to $573 \mathrm{~K}$ with slow heating rate (at $1 \mathrm{~K} / \mathrm{min}$ ) and held at this temperature for $3 \mathrm{~h}$. The tablets of catalyst were crushed and sieved into 40-60 mesh granules. The catalyst granules were loaded into an $8 \mathrm{~mm}$ tubular zirconium reactor with the volume of $14 \mathrm{ml}$. The catalyst bed was held in place by two plugs of quartz wool, and the fixed bed reactor was mounted inside a heating furnace (Tianjin Aozhan Inc.). Reactor pressure and reaction temperature were monitored by Artificial Intelligence Distributed Control System (AIDCS, YuDian Tech Co. Ltd.) Reactor pressure was controlled with a back pressure valve (Tescom 44-18) and reaction temperature was controlled by an AI-518P temperature controller (YuDian Tech Co. Ltd.). Prior to introduction of feed, the reaction system was filled with nitrogen. Upon reaching the desired reaction temperature and pressure $(453 \mathrm{~K}$ $2 \mathrm{MPa}$ ), an aqueous solution of dendroketose (10 wt\%) and organic solvent MIBK were fed to the packed tubular reactor using two HPLC pumps (Lab Alliance Series II). Dendroketose was prepared in the step before and the other chemicals were received from the supplier (Alfa Aesar). The liquid effluent was cooled in ice water and collected for quantitative analysis in a separator (Tianjin Aozhan Inc.) at ambient temperature and analyzed by HPLC.

The liquid effluent containing the MIBK solution of 4-HMF, the aqueous solution of 4-HMF and unconverted dendroketose was introduced into the continuous countercurrent extractor and extracted by pure MIBK. 4-HMF in the aqueous solution was extracted into MIBK phase and organic phase was forwarded into distillator to give the product of 4-HMF. Experimental data see Table S2.

Preparation of $\mathrm{C}_{9}-\mathrm{C}_{15}$ branched-chain alkanes from 4-HMF. Synthesis of 1,5-bis (4-(hydroxymethyl)-2-furanyl)-1,4-pentadien-3-one $\left(\mathrm{F}_{4}-\mathrm{Ac}_{-} \mathrm{F}_{4}\right)$ 1,5-bis (4-(hydroxymethyl)-2-furanyl)-1,4-pentadien-3-one $\left(\mathrm{F}_{4}-\mathrm{Ac}-\mathrm{F}_{4}\right)$ was synthesized by aldol condensation of 4-hydroxymethylfurfural (4-HMF) and acetone. 4-HMF (4.75 g; $37.7 \mathrm{mmol})$ was dissolved in 0.5 equivalents $(1.09 \mathrm{~g}, 18.85 \mathrm{mmol}$ ) of acetone and $50 \mathrm{ml}$ of $0.1 \mathrm{M} \mathrm{KOH}$. The mixture was stirred at room temperature overnight. The solid precipitate was removed by filtration and washed to neutral with water. The wet solid product could be used directly in the following step or dried in a vacuum sulfuric acid desiccator under reduce pressure overnight to provide $3.68 \mathrm{~g}(71 \%)$ of $\mathrm{F}_{4}-\mathrm{Ac}-\mathrm{F}_{4}$ as a yellow powder.

Hydrogenation of $\mathrm{F}_{4}-\mathrm{Ac}-\mathrm{F}_{4}$. The hydrogenation reaction was carried out in a stainless autoclave containing $1.2 \mathrm{~g} \mathrm{~F}_{4}-\mathrm{Ac}_{-} \mathrm{F}_{4}, 60 \mathrm{ml}$ water, and $0.24 \mathrm{~g} 5 \% \mathrm{Pd} / \mathrm{C}$ (Aldrich) at the reaction conditions of $\mathrm{P}\left(\mathrm{H}_{2}\right)=5.3 \mathrm{MPa}$ (ambient temperature) and $\mathrm{T}=393 \mathrm{~K}$ under stirring $(800 \mathrm{rpm})$. The reaction progress was measured by ${ }^{13} \mathrm{C}-\mathrm{NMR}$ spectroscopy and when no aromatic signals were detected anymore, the reaction was stopped. Then, the reactor was cooled down to room temperature, the catalyst was separated by filtration, and the filtrate was used directly in the following step.

Hydrodeoxygenation. The hydrogenation reaction was carried out in a stainless autoclave containing $9 \mathrm{ml}$ hydrogenation crude reaction mixture, $90 \mathrm{mg}$ $5 \% \mathrm{Pt} / \mathrm{C}$ (Aldrich) or $90 \mathrm{mg}$ 5\%Pd/C (Aldrich), and $30 \mathrm{mg}$ Zeolite HZSM-5 (supplied by the Catalytic Factory of Nankai University, $\mathrm{Si} / \mathrm{Al}=50$ ). After flushing the reactor with $\mathrm{H}_{2}$ for three times, reactions were conducted at 523-553 $\mathrm{K}$ in presence of $5.5 \mathrm{MPa} \mathrm{H}_{2}$ (ambient temperature) for $4 \mathrm{~h}$ with a stirring speed of $800 \mathrm{rpm}$. After cooled to ambient temperature, the organic products were extracted by hexane. The organic phase was analyzed by GC-MS and GC. Internal standard (hexadecane, TCI, purity $\geq 99.5 \%$ ) was used to determine the amount of alkanes.

Hydrogenolysis of 4-HMF. All batch reactor runs were carried out using an autoclave reactor with external temperature and stirring controller (Models 4792, Parr Instrument). For a typical hydrogenolysis run, $0.4 \mathrm{~g}$ of 4-HMF was dissolved in $10 \mathrm{ml}$ 1-butanol. Next, $0.08 \mathrm{~g}$ of pre-reduced barium promoted $\mathrm{CuCrO}_{4}$ was added to the reactor. The reactor was pressurized three times with $\mathrm{H}_{2}$ and the reaction was carried out at $493 \mathrm{~K}$ with 10 bar initial hydrogen pressure for $3 \mathrm{~h}$. After $3 \mathrm{~h}$ the reactor was cooled to room temperature, released the pressure carefully, and $0.08 \mathrm{~g} 15 \% \mathrm{Cu}$ $10 \% \mathrm{Ru} / \mathrm{C}$ was added to the reactor. The reactor was pressurized three times with $7 \mathrm{bar}$ initial hydrogen pressure and pressurized with $\mathrm{CO}_{2}$ to achieve a total system pressure of 50 bar under the specific reaction conditions, and the pressurized reactor was heated to $493 \mathrm{~K}$ for $0.5 \mathrm{~h}$. The reaction was then quenched by cooling and careful depressurization. The product solution was analyzed by HPLC and GC-MS.

1. Huber, G. W., Iborra, S. \& Corma, A. Synthesis of transportation fuels from biomass: chemistry, catalysts, and engineering. Chem. Rev. 106, 4044-4098 (2006).

2. Sanderson, K. A chewy problem. Nature 474, S12-S14 (2011).

3. Ragauskas, A. J. et al. The path forward for biofuels and biomaterials. Science 311, 484-489 (2006)

4. Chheda, J. N., Huber, G. W. \& Dumesic, J. A. Liquid-phase catalytic processing of biomass-derived oxygenated hydrocarbons to fuels and chemicals. Angew. Chem. Int. Ed. 46, 7164-7183 (2007).

5. Serrano-Ruiz, J. C. \& Dumesic, J. A. Catalytic routes for the conversion of biomass into liquid hydrocarbon transportation fuels. Energy Environ. Sci. 4, 83-99 (2011).

6. Schmidt, L. D. \& Dauenhauer, P. J. Hybrid routes to biofuels. Nature 447, 914-915 (2007)

7. Bull, T. E. Biomass in the Energy Picture. Science 285, 1209 (1999)

8. Huber, G. W., Chheda, J. N., Barrett, C. J. \& Dumesic, J. A. Production of liquid alkanes by aqueous-phase processing of biomass-derived carbohydrates. Science 308, 1446-1450 (2005).

9. Aden, A. et al., "Lignocellulosic Biomass to Ethanol Process Design and Economics Utilizing Co-Current Dilute Acid Prehydrolysis and Enzymatic Hydrolysis for Corn Stover", Report No. NREL/TP-510-32438(2002); available at (http://www.nrel.gov/docs/fy02osti/32438.pdf, accessed 22/03/2012).

10. Spath, P. L. \& Dayton, D. C. "Preliminary Screening - Technical and Economic Assessment of Synthesis Gas to Fuels and Chemicals with Emphasis on the Potential for Biomass-Derived Syngas", Report No. NREL/TP-510-34929(2003); available at (http://www.nrel.gov/docs/fy04osti/34929.pdf, accessed 22/03/2012).

11. "The Production of Methanol and Gasoline" Report of the New Zealand Institute of Chemistry; available at (http://nzic.org.nz/ChemProcesses/energy/7D.pdf, accessed 22/03/2012).

12. Moliner, M., Román-Leshkov, Y. \& Davis, M. E. Tin-containing zeolites are highly active catalysts for the isomerization of glucose in water. Proc. Natl. Acad. Sci. USA 107, 6164-6168 (2010).

13. Zhao, H., Holladay, J. E., Brown, H. \& Zhang, Z. C. Metal chlorides in ionic liquid solvents convert sugars to 5-hydroxymethylfurfural. Science 316, 1597-1600 (2007).

14. Binder, J. B. \& Raines, R. T. Simple chemical transformation of lignocellulosic biomass into furans for fuels and chemicals. J. Am. Chem. Soc. 131, 1979-1985 (2009).

15. Rosatella, A. A., Simeonov, S. P., Frade, R. F. M. \& Afonso, C. A. M. 5-Hydroxymethylfurfural (HMF) as a building block platform: Biological properties, synthesis and synthetic applications. Green Chem. 13, 754-793 (2011).

16. Nieman, J. A. \& Keay, B. A. A Facile Preparation of 2,4-Disubstituted Furans. Tetrahedron Lett. 35, 5335-5338 (1994).

17. Katritzky, A. R., Hür, D., Kirichenko, K., Ji, Y. \& Steel, P. J. Synthesis of 2, 4-disubstituted furans and 4,6-diaryl-substituted 2,3-benzo-1,3a, 6a-triazapentalenes. ARKIVOC 2004, 109-121 (2004).

18. Gerberich, H. R. \& Seaman, G. C. "Formaldehyde" in Kirk-othmer encyclopedia of chemical technology. volume 12, John Wiley and Sons, 107-128 (2007).

19. Matsumoto, T., Yamamoto, H. \& Inoue, S. Selective formation of triose from formaldehyde catalyzed by thiazolium salt. J. Am. Chem. Soc. 106, 4829-4832 (1984).

20. Avison, C. A., Dobson, I. D., Gracey, B. P. \& Kikabhia, T. Synthesis of alpha-hydroxy ketones. European patent EP0474387 (1991).

21. Avison, C. A., Dobson, I. D., Gracey, B. P., Hudson, B. \& Kikabhia, T. Production of hydroxyketones. European patent EP0480646 (1991).

22. Nagorski, R. W. \& Richard, J. P. Mechanistic imperatives for aldose-ketose isomerization in water: specific, general base- and metal ion-catalyzed isomerization of glyceraldehyde with proton and hydride transfer. J. Am. Chem. Soc. 123, 794-802 (2001).

23. Gutsche, C. D. et al. Base-catalyzed triose condensations. J. Am. Chem. Soc. 89, 1235-1245 (1967).

24. West, R. M. et al. Zeolite H-USY for the production of lactic acid and methyl lactate from C3-sugars. J. Catal. 269, 122-130 (2010).

25. Rasrendra, C. B., Fachri, B. A., Makertihartha, I. G. B. N., Adisasmito, S. \& Heeres, H. J. Catalytic conversion of dihydroxyacetone to lactic acid using metal salts in water. ChemSusChem 4, 768-777 (2011)

26. Román-Leshkov, Y., Chheda, J. N. \& Dumesic, J. A. Phase modifiers promote efficient production of hydroxymethylfurfural from fructose. Science 312, 1933 1937 (2006).

27. Yang, F., Liu, Q., Yue, M., Bai, X. \& Du, Y. Tantalum compounds as heterogeneous catalysts for saccharide dehydration to 5-hydroxymethylfurfural. Chem.Commun. 47, 4469-4471 (2011). 
28. Cook, G., Epstein, K. \& Brown, H. The Biomass Economy (Technical Report No.NREL/JA-810-31967 2002); available at (http://www.nrel.gov/docs/fy02osti/ 31967.pdf, accessed 22/03/2012).

29. Roman-Leshkov, Y., Barrett, C. J., Liu, Z. Y. \& Dumesic, J. A. Production of dimethylfuran for liquid fuels from biomass-derived carbohydrates. Nature 447, 982-985 (2007).

30. Stevens, J. G., Bourne, R. A., Twigg, M. V. \& Poliakoff, M. Real-time product switching using a twin catalyst system for the hydrogenation of furfural in supercritical CO2. Angew. Chem. Int. Ed. 49, 8856 (2010).

\section{Acknowledgements}

This work was supported by the National Basic Research Program of China 2012CB215306, the National Natural Science Foundation of China 21172209 and Chinese Academy of Science KJCX2-EW-J02.

\section{Author contributions}

J.D. and Y.F. designed experiments; J.D., T.P., Q.X. and M.-Y. C. performed the experiments; J.D., T.P., Q.-X. G. and Y.F. analyzed the data; and J.D., T.P., Q.X. Y.Z. and Y.F. wrote the paper.

\section{Additional information}

Supplementary information accompanies this paper at http://www.nature.com/ scientificreports

Competing financial interests: The University of Science and Technology of China has filed a WO provisional patent on the technology described in this manuscript.

License: This work is licensed under a Creative Commons

Attribution-NonCommercial-NoDerivs 3.0 Unported License. To view a copy of this license, visit http://creativecommons.org/licenses/by-nc-nd/3.0/

How to cite this article: Deng, J. et al. Linked strategy for the production of fuels via formose reaction. Sci. Rep. 3, 1244; DOI:10.1038/srep01244 (2013). 